\title{
Dengue Fever Outbreak Investigation and Response in Dire Dawa City Administration, Ethiopia, 2017
}

\author{
Mengistu Biru \\ Saint Paulo's Hospital Millennium Medical College, Ethiopia \\ Dessalegn Geleta* \\ Ethiopian Public Health Institute, Ethiopia \\ Neamine Tesfaye \\ Ethiopian Public Health Institute, Ethiopia \\ Mikias Aleyu \\ Ethiopian Public Health Institute, Ethiopia \\ Adamu Tayachew \\ Ethiopian Public Health Institute, Ethiopia
}

\begin{abstract}
Background: Dengue fever is caused by dengue virus (DENV), a member of the genus Flavivirus of the family Flaviviridae. The virus is transmitted by the infected female of the primary vector Aedes Aegyptus mosquitoes. Dengue fever is the world's most important re-emerging arboviral disease with over $50 \%$ of the world's population at risk of the disease. Since 2013, Ethiopia has reported more than 12,000 Dengue Fever cases. The purpose of this study is to determine risk factors of Dengue Fever outbreak and implement possible interventions in Dire Dawa City Administration

Methods: we conducted 1:2 unmatched case control study from November 1 to 15, 2017. Cases were febrile patients with two or more of the following manifestations: headache, retro orbital pain, myalgia, arthralgia, rash, hemorrhagic manifestations and leucopenia. Cases were purposively collected from health facilities and controls were people without suspected Dengue Fever symptoms. Multiple logistic regression was used to control confounders and to identify risk factors for Dengue Fever.

Results: We included 180 participants (60 cases and 120 controls), among Dengue Fever cases 46 (76.6\%) were males and $14(23.4 \%)$ were females. The entomological finding was $(\mathrm{HI}=21 \%, \mathrm{CI}=20 \%$ and $\mathrm{BI}=20 \%)$ in the study area. Presences of tree holes (adjusted OR $=8.77: 95 \% \mathrm{CI}: 2.07-37.07$ ), Uncovered window (adjusted OR $=0.09: 95 \%$ CI: $0.01-0.58$ ), not use replant (adjusted OR $=15.21: 95 \%$ CI: $3.59-54.9$ ), Not wear long sleeved clothes (adjusted OR $=19.48: 95 \%$ CI: $5.70-66.6$ ), Not sprayed in the last6mths (adjusted OR $=6.86$ : 95\% CI: 1.47 -32.02), presence of water container(adjusted OR $=15.2$ : 95\% CI: $3.33-68.8$ ) were independent risk factors for dengue fever disease. From ten serum samples, six were IGM positive for DENVII.

Conclusion: The outbreak is confirmed dengue fever outbreak and patients not using repellants, house without sprayed in the last six months, residents wear short sleeved cloths, open water containers and tree holes are significantly associated with enhancing the exposure to dengue fever disease infection.
\end{abstract}

Keywords: [Dengue fever, Diredewa, outbreak, Aedes Eagypti]

DOI: $10.7176 / \mathrm{JMPB} / 63-04$

Publication date: January $31^{\text {st }} 2020$

\section{Introduction}

Dengue Fever is caused by dengue virus (DENV), a member of the genus Flavivirus of the family Flaviviridae. There are four serotypes, DENV1 through DENV4. DENV belongs to the genus Flavivirus of family Flaviviridae $^{2}$. The virus is transmitted by the infected female of the primary vector Aedes Aegyptus mosquitoes. Infections can also be transmitted through blood transfusion, organ transplantation and possibly vertically from mother to child ${ }^{1}$.

Dengue Fever is the world's most important re-emerging arboviral disease with over $50 \%$ of the world's population at risk of the disease and 50\% residing in dengue endemic countries. Approximately 3.6 billion people are currently at risk of Dengue infection in over 100 countries of Asia, Americas and Africa. It has been estimated that 390 million dengue infections occur worldwide annually ${ }^{3}$.

Various studies hypothesized general factors to explain the reasons for the emergence of Dengue Fever. Rapid population growth, unplanned urbanization, and increased international travel in combination with vector distribution were assumed to result in extensive transmission of dengue virus in Africa ${ }^{5}$. Different categories of water storage containers can provide favorable breeding sites for Dengue Fever Vectors. Patients' reluctance to 
travel long distances for medical care and others opting for treatment from traditional healers contributed to the spread of dengue. Human settlements around an irrigation canals enhanced mosquito vector diversity. The quality of the breeding water is important to the survival of mosquito eggs ${ }^{6}$.

Since 2013, Ethiopia has reported more than 12,000 Dengue Fever cases. A confirmed Dengue Fever case was reported for the first time in Ethiopia in Dire Dawa city in mid-September 2013. Suspected cases later reported from Afar Region 2014 and Somali Region from January 2014 to June 2017 from different districts ${ }^{7}$.

Dire Dawa city administration experienced recurrent Dengue Fever outbreaks from 2013 to 2017. This outbreak was notified by the Dire Dawa City Public health emergency management Department to the national Public Health Emergency Management (PHEM) department of Ethiopian Public Health Institute on 30th October 2017.The team from Ethiopian Public Health Institute was deployed to Diredewa city administration for the outbreak investigation and response on 2nd November, 2017. Therefore, the aim of this study is to determine factors associated with Dengue Fever outbreak and implement appropriate control measure in Diredewa administration city in 2017.

\section{Material and methods \\ Study area and design}

The study was conducted at Dire Dawa Administrative city which is located in the Eastern of Addis Ababa at Latitude $9031^{\prime} \mathrm{N}$ and Longitude 410 52' E .It is about $515 \mathrm{Km}$ far from capital city of Ethiopia, Addis Ababa which is surrounded by Ethio-somali and Oromia region.

We applied descriptive data analysis of collected line list followed by facility based unmatched 1:2 case control to investigate the possible risk factor.

\section{Study Population}

Study population for this study was all residents of Diredewa city Administration who were attending the health facilities. Cases were either confirmed or epidemiologically linked dengue Fever cases while controls were all people without suspected Dengue Fever symptoms. All confirmed or epidemiologically linked cases of dengue fever found in Health facilities from November 1 to 15, 2017 were included in the study. Therefore we included 180 participants (60 cases and 120 controls) for this study.

\section{Data collection instruments and procedure}

The data was collected through face to face interview using questionnaire and document review. The questionnaire was divided in to three main areas covering demographic variables, clinical feature of the disease and risk factors associated with Dengue fever. Pre tested questionnaire were used to ensure the consistency.

For serological test, Serum samples were collected from ten cases and tested by Rapid Diagnostic Test to confirm presence of Dengue Fever Serotype (IgM). Entomological studies were conducted for 110 households that include cases and their neighbors for the presence of potential vectors. All Indoor and outdoor containers were inspected by entomologist for the presence of immature Aedes mosquito in the study area. We used three entomological indices to measure the vector infestation (House Index (HI), Container Index (CI), and the Breteau Index (BI) using the criteria of the Queensland Government $(2011)^{8}$.

House hold index $(\mathrm{HI} \%)=$ number of house hold with larva $* 100$

Among visited house hold

Container index $(\mathrm{CI} \%)=$ number of container with larva $* 100$

Among visited container

Breteau Index $(\mathrm{BI}) \quad=$ number of containers having larva*100

Number of container's visited

\section{Data Process and Analysis}

The data was entered and analyzed by using SPSS version 23 software. Descriptive statistics were used to determine the frequency of different variables. Rates, ratios and proportions were calculated. Logistic regression was applied to determine factors associated with dengue fever outbreak. Therefore, bivariate and multivariable analyses were done. Results were displayed using tables and graphs and it was interpreted using Odd ratio, $\mathrm{P}$ value $<0.05$ and $95 \%$ confidence interval.

\section{Result}

\section{Socio demographic characteristics}

Out of 60 Dengue Fever cases $46(76.6 \%)$ were males and 14(23.4\%) were females, the mean age of the cases were $24.9 \pm 8.75 \mathrm{SD}$ and the mean age for controls were $28.32 \pm 11.32 \mathrm{SD}$. From the total cases $26(43.3 \%)$ of them were farmers, $8(13 \%)$ were unemployed and $14(23.3 \%)$ of the cases were students. $51(85 \%)$ of the cases were in the age category of 15 - 45 years and $8(13 \%)$ were in the age range 5 - 15 years. 
Respondents from cases and control that are not using impregnated bed net $25(41.6 \%)$ and $29(24 \%)$ respectively. $49(81.6 \%)$ of the cases were had history of traveling to the dengue fever affected areas in the last 14 days. Additionally, 53(88.3\%) of the cases had water containing arrears (stagnant water) in the near of the household. (Table1)

Table 1: Risk factors of Dengue Fever among case and control participants in Dire Dawa City, Ethiopia, October 2017, $\mathrm{n}=180$

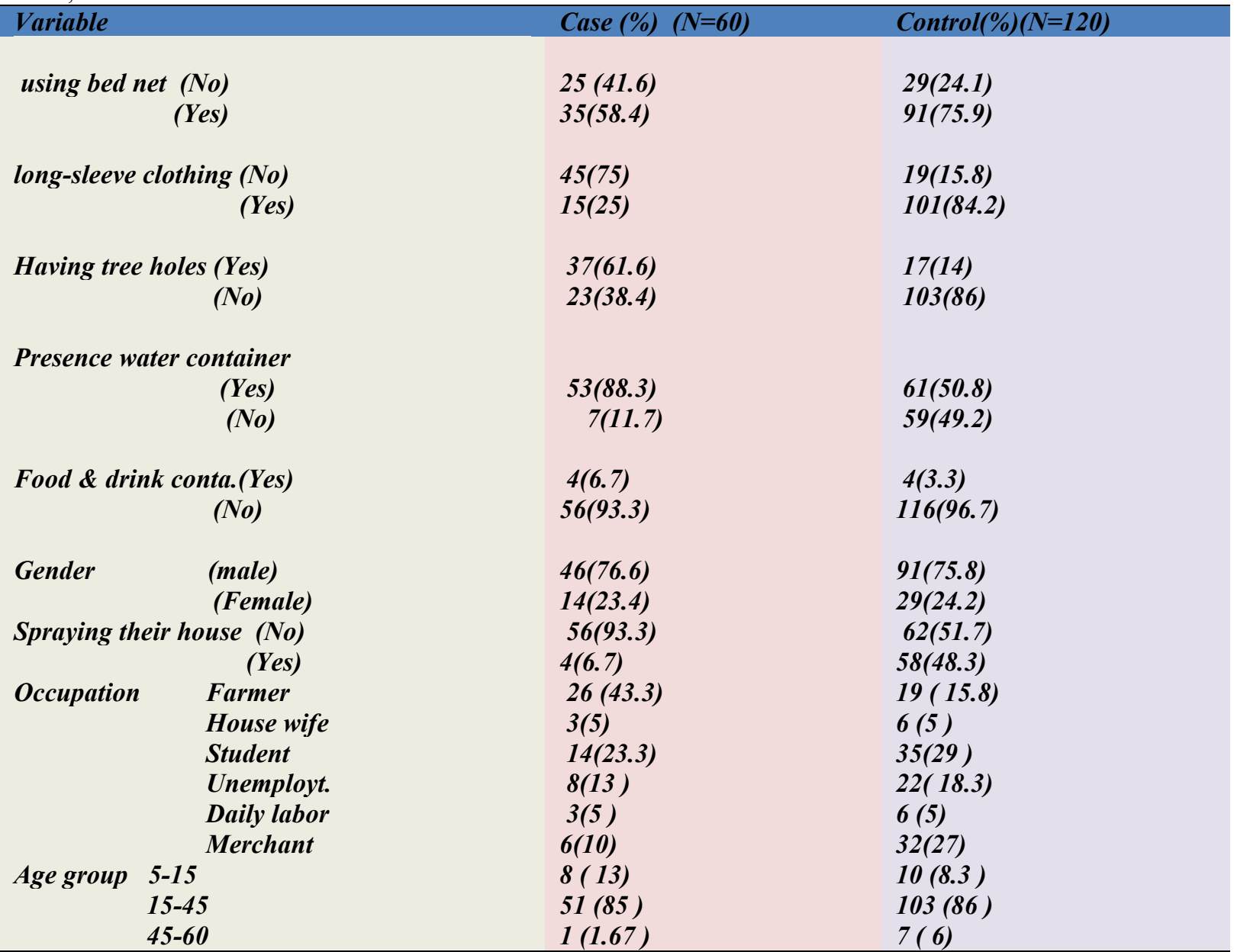

\section{Descriptive results}

A total of 309 cases were reported from study area from 07th October 2017 to 14th November 2017. After lapsed for about one week, the cases begun to be reported on the 19th October and reached its maximum record on 23rd October after which it slightly begun to bent down as shown on the below figure. (Figure1)

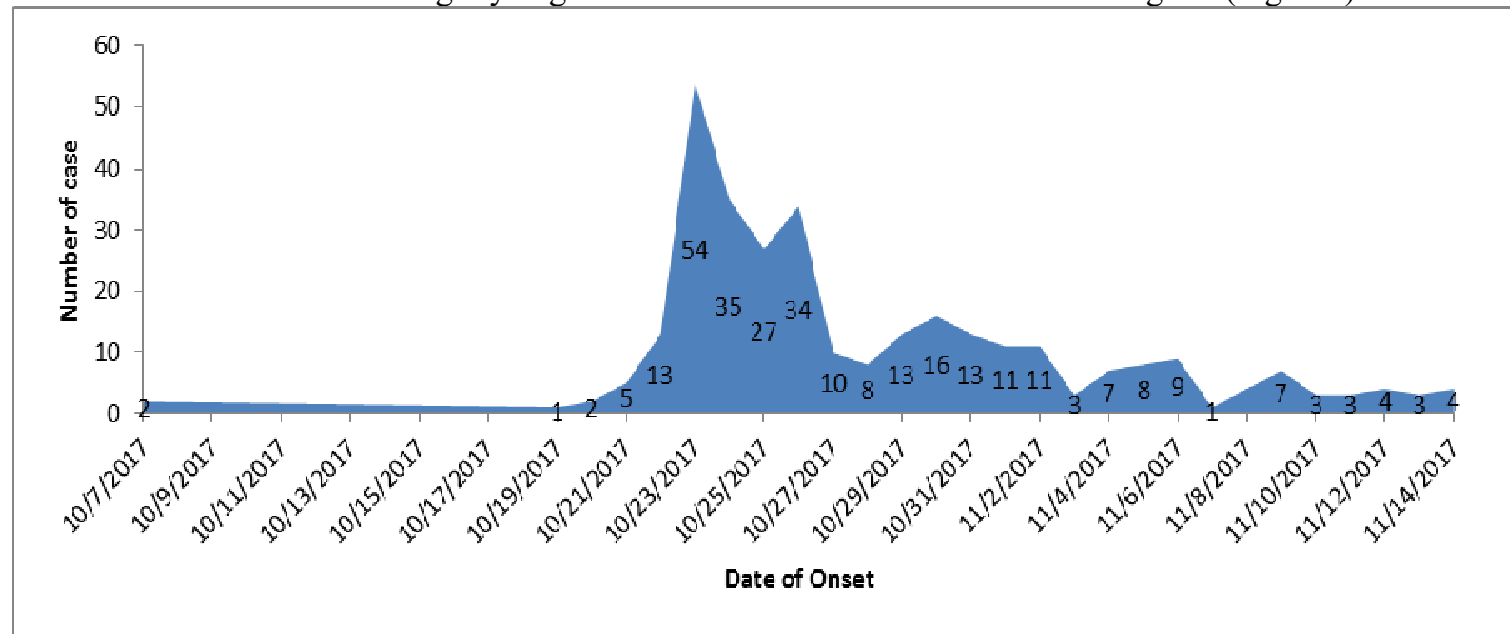

Figure 1 Epidemic curve of Dengue Fever outbreak in Dire Dawa City Administration, Ethiopia. 2017. 


\section{Discussion}

The result of this study shows all the patients diagnosed as dengue fever had $100 \%$ fever and headache in the study area. This is in consistence with the study conducted in university of Malaysia, Medical center, kuala Lumbara,finding ${ }^{4}$.

Serum samples were obtained from 10 participants for confirmation, of those six were IGM positive for DENV2 (positivity rate of $60 \%$ ). The rest of the cases were epidemiologically linked by person, place and time. The entomological investigation study revealed that $(\mathrm{HI}=21 \%, \mathrm{CI}=20 \%$ and $\mathrm{BI}=20 \%)$. According to the $\mathrm{WHO}$ criteria the area is at high risk of Dengue transmission if $\mathrm{HI}>10 \%, \mathrm{CI}>5 \%$. However, the BI shows the area is at an intermediate risk $^{8}$.

The multivariate logistic regression model showed that the risk factors were remained significant in the model: the availability of open water containers around the house, the presence of tree holes, lack of bed net utilization, house was not sprayed in the last 6 months and patients not having long sleeved cloths. (Table 2).

The model result shows those who have open water containers (stagnant water) around their houses were 15 times (adjusted OR $=15.2: 95 \% \mathrm{CI}: 3.33$-68.8) more at risk to have the disease. In consistence with this, case control study conducted in Dire Dawa during 2015 showed that people living in the areas where open water container had high morbidity rate of dengue feve ${ }^{11}$. Similar study from Vietnam identified that people living near stagnant water like ponds, lakes or open sewers had higher rates of dengue fever morbidit ${ }^{10}$.

In this study, the risk of acquiring Dengue Fever was more likely on those who did not use repellent $(\mathrm{OR}=$ 15.2; 95\% CI: 3.89 - 54.9; p-value <0.001) compared to those who used the repellents. Similar study was conducted in Lahore Pakistan city campus area of UVAS showed that patients who didn't use repellents had high risk of morbidity of dengue fever infection ${ }^{12}$. Presence of trees holes in the patio is also one of the significant factor for Dengue Infection because they serve as breeding site for the Aedes mosquito. In this study presence of trees holes in the compound significantly associated with Dengue Fever infection (OR=8.77; 95\%CI: 2.07 37.07; P-value $=0.003)$. in consistence with this, study conducted in Brazil showed plants with temporary water pools in the garden were a risk factor for Dengue infection ${ }^{9}$.

This study also revealed that participants not wearing long sleeved cloths $(\mathrm{OR}=19.48 ; 95 \% \mathrm{CI} ; 5.70-66.6$; P-value $<0.001)$ had 20 times the risk of acquiring Dengue Fever infection as compared to the users. Similar study was conducted in Diredewa in 2015 was shows that there has association between the wearing type of cloth and the risk of acquiring the Dengue Fever infection ${ }^{11}$.

In a complex epidemiological setting, transmission dynamics involve interactions among people, Dengue viruses, vectors and ecosystems, where biotic and abiotic determinants have both direct and indirect influences on transmission. Our model did not account for all possible variables. More information is needed to further unravel the predictors of dengue transmission in Diredewa city administration, which will help to inform planning of prevention and control measures.

Table 2: Multivariable logistic regression model for significant risk factors $(\mathrm{p}<0.05)$ of Dengue Fever in Dire Dewa city, October 2017, Ethiopia

\begin{tabular}{|c|c|c|c|}
\hline Characteristics & P-Values & S.E & AOR(95\%CI) \\
\hline Presence of tree holes & 0.003 & 0.73 & $8.77(2.07-37.07)$ \\
\hline Not use replant & 0.000 & 0.695 & $15.21(3.89-54.9)$ \\
\hline No long sleeved cloth & 0.000 & 0.626 & $19.48(5.70-66.6)$ \\
\hline Not sprayed in the last6mths & 0.014 & 0.785 & $6.86(1.47-32.02)$ \\
\hline Open water container & 0.000 & 0.770 & $15.2(3.33-68.8)$ \\
\hline
\end{tabular}

\section{SE, Standard Error; AOR, Adjusted Odds Ratio}

\section{Conclusion}

This study was conducted to find association between Dengue fever and associated risk factors among the residents of Dire Dawa city administration, Ethiopia. There are several factors like not using repellants, house is not sprayed in the last six months, residents hadn't wear long sleeved cloths, the presence of open water containers and the presence of tree holes, which are significantly associated with enhancing the exposure to dengue fever disease infection. The outbreak is ongoing in the study area during the investigation and intervention period.

\section{Abbreviations}

AR, Attack Rate; CFR, Case Fatality Rate; DENV, Dengue Virus; DHF, Dengue Hemorrhagic Fever; EPHI, Ethiopian Public Health Institute; IGM, Immune globulin M; PHEM, Public health Emergency Management; RDT, Rapid Diagnostic Test 


\section{Ethical considerations}

Written letter was submitted to Ethiopian Public Health Institute (EPHI), Public Health Emergency Management Center (cPHEM) and permission to investigate the outbreak was obtained both in EPHI and Dire Dawa city administration health bureau. Permission was obtained from the health facilities administration before approaching the patients. After verbal informed consent was given to patient, each case was interviewed at the hospital, their houses were visited and neighbors were interviewed.

\section{Data Sharing Statement}

The datasets used and/or analyzed during the current study are available from the first author on reasonable request.

\section{Acknowledgments}

We would like to express our heartfelt gratitude to study participants, data collectors and supervisors. Moreover, we would like to give our appreciation to Ethiopian Public Health Institute and Dire Dawa City Admnistration Health Bureau for their effort and ongoing cooperation for the effectiveness of this study.

\section{Authors Contribution}

TB was involved in conception of the study. DG was significantly involved in the design, selection of articles, and manuscript preparation and revision. NT, MA, TG and AT and the first authors contributed to data analysis, drafting or revising the article, gave final approval of the version to be published and agreed to be accountable for all aspects of the work.

\section{Disclosure}

The authors report no conflicts of interest in this work.

\section{References}

1. Eldigail mh, adam gk, babiker ra, khalid $\mathrm{f}$, adam ia, omer oh, et al. Prevalence of dengue fever virus antibodies and associated risk factors among residents of el-gadarif state , sudan. 2018;1-8.

2. Lew rj, tsai w, wang w. Dengue outbreaks in hawai ' $\mathrm{i}$ after wwii - a review of public health response and scientific literature. 2018;77(12):315-8.

3. Lutomiah j, barrera r, makio a, mutisya j, koka h, owaka s, et al. Dengue outbreak in mombasa city, kenya , 2013 - 2014 : entomologic investigations. 2016;2013-4.

4. Ibrahim j, nissapatorn v. Retrospective study of dengue fever ( df ) and dengue hemorrhagic fever ( dhf ) patients at university malaya medical center, kuala lumpur, malaysia in the year 2005 retrospective study of dengue fever ( $\mathrm{df}$ ) and dengue hemorrhagic fever ( dhf ) patients at university malaya medical center, kuala lumpur, malaysia in the year 2005. 2007; (june 2014).

5. Woyessa a. The emergence of dengue fever in ethiopia. 1960;

6. Ismail $\mathrm{s}$, zulkifli $\mathrm{m}$, mansor $\mathrm{r}$, mat yusof $\mathrm{m}$, ismail mi. The role of exploratory data analysis (eda) in electricity forecasting. Pertanika j soc sci humanit. 2016;24(october):93-100.

7. Ahmed ym, salah aa. Epidemiology of dengue fever in ethiopian somali region : retrospective health facility based study email address : 2016;2(2):51-6.

8. Programme s, diseases t. Review of entomological sampling methods and indicators for.

9. Heukelbach è, oliveira âs de, kerr-pontes ârs, feldmeier h. Risk factors associated with an outbreak of dengue fever in a favela in fortaleza, north-east brazil. 2001;6(8):635-42.

10. Toan dtt, hoat $\mathrm{ln}$, hu w, wright $\mathrm{p}$. Risk factors associated with an outbreak of dengue fever / dengue haemorrhagic fever in hanoi , vietnam. 2019;(2015):1594-8.

11. Degife $\mathrm{lh}$, worku y, belay d, bekele a, hailemariam z. Factors associated with dengue fever outbreak in dire dawa administration city , october , 2015 , Ethiopia - case control study. 2019;1-7.

12. Chaudhry $\mathrm{m}$, ahmad $\mathrm{s}$, nisar $\mathrm{m}$, rashid h bin, mushtaq $\mathrm{mh}$, ijaz $\mathrm{t}$, et al. A matched case - control study to identify potential risk factors of dengue fever among residents of a local university, lahore. 2015;21(3):173-7. 